\title{
La ciencia lo reafirma: el cambio climático es real
}

Climate Scientists Virtually Unanimous:

Anthropogenic Global Warming Is True

Powell, James Lawrence

Tallahassee, Florida, 32306-4453, USA

Department of Educational Psychology

and Learning Systems

Florida State University

Bulletin of Science, Technology \& Society

2016

Páginas 121-124

DOI 10.1177/0270467616634958

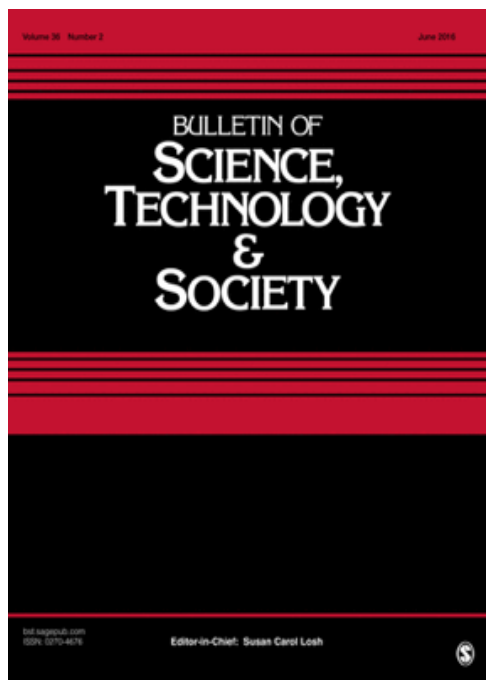

Reseñado por Efraín Bámaca-López, doctorando del programa de Ciencia, Tecnología y Sociedad en la Universidad Federal de São Carlos (UFSCar), Brasil. eefrain@ufscar.br

El cambio climático es un hecho real, el consenso científico lo confirma, y con una gran probabilidad es el ser humano el único gran responsable (IPCC, 2007, 2014; Stocker et al., 2013). Dicho artículo suma argumentos a los aportes ya conocidos en los informes del Intergovernmental Panel on Climate Change (IPCC). La novedad del mismo radica en ir más allá de la actual frontera del conocimiento sobre el análisis a la literatura científica en torno al tema del cambio climático.

El artículo se presenta seccionado en una introducción, un apartado en relación a los estudios previos sobre el hecho del cambio climático en la literatura científica, con especial atención a los resultados presentados por Cook et al (2013) en el que se hace un análisis de los artículos científicos publicados del año 1991 hasta el año 2011: "Cook et al. reported that, "Among abstracts expressing a position [emphasis added] on AGW, 97.1\% endorsed the consensus position that humans are causing global warming." (p. 122). Posteriormente hace alusión al consenso en la literatura científica con el planteamiento de tres ejemplos históricos (continental drift, meteorite impact, and global warming) en donde por décadas no se tuvo comprobación científica que permitiera creer lo contrario, por ahora con el Anthropogenic Global Warming no hay evidencia convincente en contra, puesto que como él mismo afirma: "If such evidence existed, we would already know about it and not have to conduct a needle in the haystack search in a vain attempt to find it" (p. 124). Posteriormente anota cuales fueron sus palabras claves para la búsqueda de sus artículos objeto de estudio "global warming or global climate change or climate change" (p. 123). En base a dicho análisis deja claro en su conclusión que la existencia del calentamiento global es un hecho causado por el hombre.

Las corrientes negacionistas del cambio climático también encuentran eco en la literatura científica, y el artículo no omite a los cuatros autores que hablaron del hecho: 
During 2013 and 2014, only 4 of 69,406 authors of peer-reviewed articles on global warming, $0.0058 \%$ or 1 in 17,352, rejected AGW (Anthropogenic Global Warming). Thus, the consensus on AGW among publishing scientists is above $99.99 \%$, verging on unanimity (p.121). Avakyan (2013a, 2013b), Gervais (2014), Happer (2014), and Hug (2013). These represent a proportion of 1 article in 4,842 or $0.021 \%$. With regard to the authors, 4 reject AGW: 1 in 17,352 or $0.0058 \%$. As explained, I interpret this to mean that $99.99 \%$ of publishing scientists accept $A G W$ : virtual unanimity (p. 124).

El presente artículo es altamente recomendado para comunicadores dedicados a la ciencia y con especial atención al tema del cambio climático, no por ello deja de ser sugestivo para todo investigador en general, tomadores de decisiones, estudiantes de posgraduación tanto a nivel de maestría como de doctorado, medios de comunicación, sociedad civil y todo aquel ciudadano que sienta motivación por conocer sobre el hecho del cambio climático. Climate Scientists Virtually Unanimous: Anthropogenic Global Warming Is True oferece un buen análisis bibliométrico en torno a la información surtida en revistas científicas sobre el cambio climático, la lectura es altamente recomendada.

\section{REFERENCIAS}

- Avakyan, S. V. (2013a). Problems of climate as a problem of optics. Journal of Optical Technology, 80, 717-721.

- Avakyan, S. V. (2013b). The role of solar activity in global warm- ing. Herald of the Russian Academy of Sciences, 83, 275-285.

- Cook, J., Nuccitelli, D., Green, S. A., Richardson, M., Winkler, B., Painting, R., ... \& Skuce, A. (2013). Quantifying the consensus on anthropogenic global warming in the scientific literature. Environmental research letters, 8(2), 024024.
- Gervais, F. (2014). Tiny warming of residual anthropogenic CO2. International Journalof Modern Physics B, 28, 1450095.

- Happer, W. (2014). Why has global warming paused? International Journal of Modern Physics A, 29, 1460003.

- Hug, H. (2013). "The Climate Models are inadequate": Heinz Hug queries the significance of $\mathrm{CO} 2$ for climate change. Nachrichten Aus Der Chemie, 61, 132.

- IPCC. (2007). Cambio climático 2007: informe de síntesis. Contribución de los Grupos de trabajo I, II y III al Cuarto Informe de evaluación del Grupo Intergubernamental de Expertos sobre el Cambio Climático (Informe de síntesis). Intergovernmental Panel on Climate Change. Ginebra: Intergovernmental Panel on Climate Change.

- IPCC. (2014). Cambio climático 2014: Informe de Síntesis. (Equipo principal de

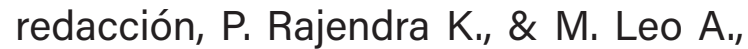
Eds.)Cambio climático 2014: Informe de síntesis. Contribución de los Grupos de trabajo I, II y III al Quinto Informe de Evaluación del Grupo Intergubernamental de Expertos sobre el Cambio Climático. Ginebra, Suiza.

- Stocker, T.F., D. Qin, G.-K. Plattner, L.V. Alexander, S.K. Allen, N.L. Bindoff, F.-M. Bréon, J.A. Church, U. Cubasch, S. Emori, P. Forster, P. Friedlingstein, N. Gillett, J.M. Gregory, D.L. Hartmann, E. Jansen, B. Kirtman, R. Knutti, K. Krishna Kumar, P., D. G. V. y S.-P. X. (2013). Resumen técnico. En V. B. y P. M. M. Stocker, T.F., D. Qin, G.-K. Plattner, M. Tignor, S.K. Allen, J. Boschung, A. Nauels, Y. Xia (Ed.), Cambio climático 2013. Bases físicas. Contribución del Grupo de trabajo I al Quinto Informe de Evaluación del Grupo Intergubernamental de Expertos sobre el Cambio Climático (p. 182). Informe de síntesis, Cambridge y Nueva York, Reino Unido y Estados Unidos de América: Cambridge University Press. 